\title{
Exact Solutions of the Space Time Fractional Symmetric Regularized Long Wave Equation Using Different Methods
}

\author{
Özkan Güner ${ }^{1}$ and Dursun Eser ${ }^{2}$ \\ ${ }^{1}$ Department of Management Information Systems, School of Applied Sciences, Dumlupinar University, 43100 Kutahya, Turkey \\ ${ }^{2}$ Department of Mathematics-Computer, Art-Science Faculty, Eskisehir Osmangazi University, 26480 Eskisehir, Turkey
}

Correspondence should be addressed to Dursun Eser; deser@ogu.edu.tr

Received 4 April 2014; Accepted 22 June 2014; Published 22 July 2014

Academic Editor: Hossein Jafari

Copyright (C) 2014 Ö. Güner and D. Eser. This is an open access article distributed under the Creative Commons Attribution License, which permits unrestricted use, distribution, and reproduction in any medium, provided the original work is properly cited.

We apply the functional variable method, exp-function method, and $\left(G^{\prime} / G\right)$-expansion method to establish the exact solutions of the nonlinear fractional partial differential equation (NLFPDE) in the sense of the modified Riemann-Liouville derivative. As a result, some new exact solutions for them are obtained. The results show that these methods are very effective and powerful mathematical tools for solving nonlinear fractional equations arising in mathematical physics. As a result, these methods can also be applied to other nonlinear fractional differential equations.

\section{Introduction}

Fractional calculus is a field of mathematics that grows out of the traditional definitions of calculus. Fractional calculus has gained importance during the last decades mainly due to its applications in various areas of physics, biology, mathematics, and engineering. Some of the current application fields of fractional calculus include fluid flow, dynamical process in self-similar and porous structures, electrical networks, probability and statistics, control theory of dynamical systems, systems identification, acoustics, viscoelasticity, control theory, electrochemistry of corrosion, chemical physics, finance, optics, and signal processing [1-3].

There are several definitions of the fractional derivative which are generally not equivalent to each other. Some of these definitions are Sun and Chen's fractal derivative $[4,5]$, Cresson's derivative [6, 7], Grünwald-Letnikov's fractional derivative [8], Riemann-Liouville's derivative [8], and Caputo's fractional derivative [9]. But the Riemann-Liouville derivative and the Caputo derivative are the most used ones.

Lately, both mathematicians and physicists have devoted considerable effort to the study of explicit solutions to nonlinear fractional differential equations. Many powerful methods have been presented. Among them are the fractional $\left(G^{\prime} / G\right)$-expansion method [10-13], the fractional exp-function method [14-16], the fractional first integral method [17, 18], the fractional subequation method [19-22], the fractional functional variable method [23], the fractional modified trial equation method [24, 25],andthe fractional simplest equation method [26].

The paper suggests the functional variable method, the exp-function method, the $\left(G^{\prime} / G\right)$-expansion method, and fractional complex transform to find the exact solutions of nonlinear fractional partial differential equation with the modified Riemann-Liouville derivative.

This paper is organized as follows. In Section 2, basic definitions of Jumarie's Riemann-Liouville derivative are given; in Section 3, description of the methods for FDEs is given. Then, in Section 4, these methods have been applied to establish exact solutions for the space-time fractional symmetric regularized long wave (SRLW) equation. Conclusion is given in Section 5.

\section{Jumarie's Modified Riemann-Liouville Derivative}

Recently, a new modified Riemann-Liouville derivative is proposed by Jumarie $[27,28]$. This new definition of fractional derivative has two main advantages: firstly, comparing 
with the Caputo derivative, the function to be differentiated is not necessarily differentiable; secondly, different from the Riemann-Liouville derivative, Jumarie's modified RiemannLiouville derivative of a constant is defined to be zero. Jumarie's modified Riemann-Liouville derivative of order $\alpha$ is defined by

$$
\begin{aligned}
& D_{x}^{\alpha} f(x) \\
& =\left\{\begin{array}{c}
\frac{1}{\Gamma(1-\alpha)} \\
\times \frac{d}{d x} \int_{0}^{x}(x-\xi)^{-\alpha}(f(\xi)-f(0)) d \xi, \\
\left(f^{(n)}(x)\right)^{(\alpha-n)}, \quad n \leq \alpha<n+1, n \geq 1,
\end{array}\right.
\end{aligned}
$$

where $f: R \rightarrow R, x \rightarrow f(x)$ denotes a continuous (but not necessarily first-order-differentiable) function. Some useful formulas and results of Jumarie's modified Riemann-Liouville derivative can be found in $[28,29]$

$$
\begin{gathered}
D_{x}^{\alpha} x^{r}=\frac{\Gamma(1+r)}{\Gamma(1+r-\alpha)} x^{r-\alpha}, \\
D_{x}^{\alpha}(u(x) v(x))=v(x) D_{x}^{\alpha} u(x)+u(x) D_{x}^{\alpha} v(x), \\
D_{x}^{\alpha} f[u(x)]=f_{u}^{\prime}(u) D_{x}^{\alpha} u(x), \\
D_{x}^{\alpha} f[u(x)]=D_{u}^{\alpha} f(u)\left(u^{\prime}(x)\right)^{\alpha},
\end{gathered}
$$

which are direct consequences of the equality

$$
\Gamma(1+\alpha) d x=d^{\alpha} x
$$

In the above formulas (3)-(5), $u(x)$ is nondifferentiable function in (3) and (4) and differentiable in (5). The function $v(x)$ is nondifferentiable, and $f(u)$ is differentiable in (4) and nondifferentiable in (5). Because of these, the formulas (3)(5) should be used carefully. The above equations play an important role in fractional calculus in Sections 3 and 4.

\section{Description of the Methods for FDEs}

We consider the following general nonlinear FDEs of the type

$$
\begin{array}{r}
P\left(u, D_{t}^{\alpha} u, D_{x}^{\beta} u, D_{y}^{\psi}, D_{t}^{\alpha} D_{t}^{\alpha} u, D_{t}^{\alpha} D_{x}^{\beta} u,\right. \\
\left.D_{x}^{\beta} D_{x}^{\beta} u, D_{x}^{\beta} D_{y}^{\psi} u, D_{y}^{\psi} D_{y}^{\psi} u, \ldots\right)=0, \\
0<\alpha, \beta, \psi<1,
\end{array}
$$

where $u$ is an unknown function. $P$ is a polynomial of $u$ and its partial fractional derivatives, in which the highest order derivatives and the nonlinear terms are involved.

The fractional complex transform [30-32] is the simplest approach to convert the fractional differential equations into ordinary differential equations. This makes the solution procedure extremely simple. The traveling wave variable is

$$
u(x, y, t)=U(\xi)
$$

where

$$
\xi=\frac{\tau x^{\beta}}{\Gamma(1+\beta)}+\frac{\delta y^{\psi}}{\Gamma(1+\psi)}+\frac{\lambda t^{\alpha}}{\Gamma(1+\alpha)},
$$

where $\tau, \delta$, and $\lambda$ are nonzero arbitrary constants. We can rewrite $(7)$ in the following nonlinear ODE:

$$
Q\left(U, U^{\prime}, U^{\prime \prime}, U^{\prime \prime \prime}, \ldots\right)=0,
$$

where the prime denotes the derivation with respect to $\xi$. Now we consider three different methods.

3.1. Basic Idea of Functional Variable Method. The features of this method are presented in [33]. We describe functional variable method to find exact solutions of nonlinear spacetime fractional differential equations as follows.

Let us make a transformation in which the unknown function $U$ is considered as a functional variable in the form

$$
U_{\xi}=F(U)
$$

and some successive derivatives of $U$ are

$$
\begin{aligned}
& U_{\xi \xi}=\frac{1}{2}\left(F^{2}\right)^{\prime}, \\
& U_{\xi \xi \xi}=\frac{1}{2}\left(F^{2}\right)^{\prime \prime} \sqrt{F^{2}}, \\
& U_{\xi \xi \xi \xi}=\frac{1}{2}\left[\left(F^{2}\right)^{\prime \prime \prime} F^{2}+\left(F^{2}\right)^{\prime \prime}\left(F^{2}\right)^{\prime}\right],
\end{aligned}
$$

where "'" stands for $d / d U$. The ODE (10) can be reduced in terms of $U, F$, and its derivatives by using the expressions of (12) into (10) as

$$
R\left(U, F, F^{\prime}, F^{\prime \prime}, F^{\prime \prime \prime}, F^{(4)}, \ldots\right)=0 .
$$

The key idea of this particular form (13) is of special interest since it admits analytical solutions for a large class of nonlinear wave type equations. Integrating (13) gives the expression of $F$. This and (11) give the appropriate solutions to the original problem.

3.2. Basic Idea of Exp-Function Method. According to expfunction method, developed by $\mathrm{He}$ and Abdou [34], we assume that the wave solution can be expressed in the following form:

$$
U(\xi)=\frac{\sum_{n=-c}^{d} a_{n} \exp [n \xi]}{\sum_{m=-p}^{q} b_{m} \exp [m \xi]},
$$


where $p, q, c$, and $d$ are positive integers which are known to be further determined and $a_{n}$ and $b_{m}$ are unknown constants. We can rewrite (14) in the following equivalent form:

$$
U(\xi)=\frac{a_{-c} \exp [-c \xi]+\cdots+a_{d} \exp [d \xi]}{b_{-p} \exp [-p \xi]+\cdots+b_{q} \exp [q \xi]}
$$

This equivalent formulation plays an important and fundamental part in finding the analytic solution of problems. To determine the value of $c$ and $p$, we balance the linear term of highest order of (10) with the highest order nonlinear term. Similarly, to determine the value of $d$ and $q$, we balance the linear term of lowest order of (10) with lowest order nonlinear term [35-40].

3.3. Basic Idea of $\left(G^{\prime} / G\right)$-Expansion Method. According to $\left(G^{\prime} / G\right)$-expansion method, developed by Wang et al. [41], the solution of (10) can be expressed by a polynomial in $\left(G^{\prime} / G\right)$ as

$$
U(\xi)=\sum_{i=0}^{m} a_{i}\left(\frac{G^{\prime}}{G}\right)^{i}, \quad a_{m} \neq 0,
$$

where $a_{i}(i=0,1,2, \ldots, m)$ are constants, while $G(\xi)$ satisfies the following second-order linear ordinary differential equation:

$$
G^{\prime \prime}(\xi)+\lambda G^{\prime}(\xi)+\mu G(\xi)=0,
$$

where $\lambda$ and $\mu$ are constants. The positive integer $m$ can be determined by considering the homogeneous balance between the highest order derivatives and the nonlinear terms appearing in (10). By substituting (16) into (10) and using (17) we collect all terms with the same order of $\left(G^{\prime} / G\right)$. Then by equating each coefficient of the resulting polynomial to zero, we obtain a set of algebraic equations for $a_{i}(i=$ $0,1,2, \ldots, m), \lambda, \mu, \tau, \delta$ and $\lambda$. Finally solving the system of equations and substituting $a_{i}(i=0,1,2, \ldots, m), \lambda, \mu, \tau, \delta$, $\lambda$, and the general solutions of (17) into (16), we can get a variety of exact solutions of (7) $[42,43]$.

\section{Exact Solutions of Space-Time Fractional Symmetric Regularized Long Wave (SRLW) Equation}

We consider the space-time fractional symmetric regularized long wave (SRLW) equation [44]

$$
\begin{array}{r}
D_{t}^{2 \alpha} u+D_{x}^{2 \alpha} u+u D_{t}^{\alpha}\left(D_{x}^{\alpha} u\right) \\
+D_{x}^{\alpha} u D_{t}^{\alpha} u+D_{t}^{2 \alpha}\left(D_{x}^{2 \alpha} u\right)=0, \\
0<\alpha \leq 1
\end{array}
$$

which arises in several physical applications including ion sound waves in plasma. For $\alpha=1$, it is shown that this equation describes weakly nonlinear ion acoustic and space-charge waves, and the real-valued $u(x, t)$ corresponds to the dimensionless fluid velocity with a decay condition [45].

We use the following transformations:

$$
\begin{gathered}
u(x, t)=U(\xi), \\
\xi=\frac{k x^{\alpha}}{\Gamma(1+\alpha)}+\frac{c t^{\alpha}}{\Gamma(1+\alpha)},
\end{gathered}
$$

where $k$ and $c$ are nonzero constants.

Substituting (20) with (1) into (18), equation (18) can be reduced into an ODE:

$$
\left(c^{2}+k^{2}\right) U^{\prime \prime}+c k U U^{\prime \prime}+c k\left(U^{\prime}\right)^{2}+c^{2} k^{2} U^{\prime \prime \prime \prime}=0,
$$

where " $U^{\prime \prime}=d U / d \xi$.

4.1. Exact Solutions by Functional Variable Method. Integrating (21) twice and setting the constants of integration to be zero, we obtain

$$
\left(c^{2}+k^{2}\right) U+c k \frac{U^{2}}{2}+c^{2} k^{2} U^{\prime \prime}=0
$$

or

$$
U_{\xi \xi}=-\frac{c^{2}+k^{2}}{c^{2} k^{2}} U-\frac{U^{2}}{2 c k}
$$
(22) to

Then we use the transformation (11) and (12) to convert

$$
\begin{aligned}
& \frac{1}{2}\left(F^{2}\right)^{\prime}=-\frac{c^{2}+k^{2}}{c^{2} k^{2}} U-\frac{U^{2}}{2 c k}, \\
& F(U)=\mp U \sqrt{-\frac{c^{2}+k^{2}}{c^{2} k^{2}}-\frac{U}{3 c k}} .
\end{aligned}
$$

The solution of (21) is obtained as

$$
U(\xi)=-\frac{3\left(c^{2}+k^{2}\right)}{c k} \sec ^{2}\left(\frac{\sqrt{c^{2}+k^{2}}}{2 k c} \xi\right)
$$

So we have

$$
\begin{aligned}
u_{1}(x, t)= & -\frac{3\left(c^{2}+k^{2}\right)}{c k} \\
& \times \sec ^{2}\left\{\frac{\sqrt{c^{2}+k^{2}}}{2 k c}\left(\frac{k x^{\alpha}}{\Gamma(1+\alpha)}+\frac{c t^{\alpha}}{\Gamma(1+\alpha)}\right)\right\},
\end{aligned}
$$

which is the exact solution of space-time fractional symmetric regularized long wave (SRLW) equation. One can see that the result is different than results of Alzaidy [44]. 
4.2. Exact Solutions by Exp-Function Method. Balancing the order of $U^{\prime \prime}$ and $U^{2}$ in (22), we obtain

$$
\begin{aligned}
U^{\prime \prime} & =\frac{c_{1} \exp [-(c+3 p) \xi]+\cdots}{c_{2} \exp [-4 p \xi]+\cdots}, \\
U^{2} & =\frac{c_{3} \exp [-2 c \xi]+\cdots}{c_{4} \exp [-2 p \xi]+\cdots}
\end{aligned}
$$

where $c_{i}$ are determined coefficients only for simplicity. Balancing highest order of exp-function in (27) we have

$$
-(c+3 p)=-(2 c+2 p)
$$

which leads to the result:

$$
p=c
$$

In the same way, we balance the linear term of the lowest order in (22), to determine the values of $d$ and $q$

$$
\begin{gathered}
U^{\prime \prime}=\frac{\cdots+d_{1} \exp [(d+3 q) \xi]}{\cdots+d_{2} \exp [4 q \xi]}, \\
U^{2}=\frac{\cdots+d_{3} \exp [2 d \xi]}{\cdots+d_{4} \exp [2 q \xi]},
\end{gathered}
$$

where $d_{i}$ are determined coefficients only for simplicity. From (30), we have

$$
3 q+d=2 d+2 q
$$

and this gives

$$
q=d
$$

For simplicity, we set $p=c=1$ and $q=d=1$, so (15) reduces to

$$
U(\xi)=\frac{a_{1} \exp (\xi)+a_{0}+a_{-1} \exp (-\xi)}{b_{1} \exp (\xi)+b_{0}+b_{-1} \exp (-\xi)}
$$

Substituting (33) into (22) and using Maple, we obtain

$$
\begin{aligned}
& \frac{1}{A}\left[R_{3} \exp (3 \xi)+R_{2} \exp (2 \xi)+R_{1} \exp (\xi)+R_{0}\right. \\
& \left.\quad+R_{-1} \exp (-\xi)+R_{-2} \exp (-2 \xi)+R_{-3} \exp (-3 \xi)\right]=0
\end{aligned}
$$

where

$$
\begin{aligned}
& A=\left(b_{-1} \exp (-\xi)+b_{0}+b_{1} \exp (\xi)\right)^{3}, \\
& R_{3}=k^{2} a_{1} b_{1}^{2}+c^{2} a_{1} b_{1}^{2}+\frac{1}{2} c k a_{1}^{2} b_{1}, \\
& R_{2}=k^{2} a_{0} b_{1}^{2}+c^{2} a_{0} b_{1}^{2}-c^{2} k^{2} a_{1} b_{1} b_{0}+c k a_{1} a_{0} b_{1} \\
& +2 c^{2} a_{1} b_{1} b_{0}+\frac{1}{2} c k a_{1}^{2} b_{0} \\
& +c^{2} k^{2} a_{0} b_{1}^{2}+2 k^{2} a_{1} b_{1} b_{0} \\
& R_{1}=2 k^{2} a_{0} b_{1} b_{0}+c^{2} a_{1} b_{0}^{2}+c^{2} a_{-1} b_{1}^{2}+k^{2} a_{-1} b_{1}^{2} \\
& -c^{2} k^{2} a_{0} b_{1} b_{0}+c k a_{1} a_{-1} b_{1}-4 c^{2} k^{2} a_{1} b_{1} b_{-1} \\
& +c k a_{1} a_{0} b_{0}+k^{2} a_{1} b_{0}^{2}+2 c^{2} a_{0} b_{1} b_{0}+\frac{1}{2} c k a_{0}^{2} b_{1} \\
& +2 k^{2} a_{1} b_{1} b_{-1}+c^{2} k^{2} a_{1} b_{0}^{2}+4 c^{2} k^{2} a_{-1} b_{1}^{2} \\
& +2 c^{2} a_{1} b_{1} b_{-1}+\frac{1}{2} c k a_{1}^{2} b_{-1} \\
& R_{0}=2 k^{2} a_{0} b_{1} b_{-1}+2 c^{2} a_{-1} b_{1} b_{0}+2 k^{2} a_{1} b_{0} b_{-1} \\
& +2 k^{2} a_{-1} b_{1} b_{0}+2 c^{2} a_{1} b_{0} b_{-1}+2 c^{2} a_{0} b_{1} b_{-1} \\
& +3 c^{2} k^{2} a_{-1} b_{0} b_{1}+c k a_{1} a_{-1} b_{0}+c k a_{0} a_{-1} b_{1} \\
& +3 c^{2} k^{2} a_{1} b_{0} b_{-1}+\frac{1}{2} c k a_{0}^{2} b_{0}-6 c^{2} k^{2} a_{0} b_{1} b_{-1} \\
& +k^{2} a_{0} b_{0}^{2}+c^{2} a_{0} b_{0}^{2}+c k a_{1} a_{0} b_{-1} \\
& R_{-1}=k^{2} a_{1} b_{-1}^{2}+c^{2} a_{1} b_{-1}^{2}+c^{2} a_{-1} b_{0}^{2}+k^{2} a_{-1} b_{0}^{2} \\
& -4 c^{2} k^{2} a_{-1} b_{1} b_{-1}-c^{2} k^{2} a_{0} b_{-1} b_{0}+c k a_{1} a_{-1} b_{-1} \\
& +c k a_{0} a_{-1} b_{0}+\frac{1}{2} c k a_{-1}^{2} b_{1}+2 k^{2} a_{-1} b_{1} b_{-1} \\
& +2 k^{2} a_{0} b_{0} b_{-1}+c^{2} k^{2} a_{-1} b_{0}^{2}+\frac{1}{2} c k a_{0}^{2} b_{-1} \\
& +2 c^{2} a_{0} b_{0} b_{-1}+2 c^{2} a_{-1} b_{1} b_{-1}+4 c^{2} k^{2} a_{1} b_{-1}^{2}, \\
& R_{-2}=k^{2} a_{0} b_{-1}^{2}+c^{2} a_{0} b_{-1}^{2}-c^{2} k^{2} a_{-1} b_{0} b_{-1}+c k a_{0} a_{-1} b_{-1} \\
& +\frac{1}{2} c k a_{-1}^{2} b_{0}+c^{2} k^{2} a_{0} b_{-1}^{2}+2 c^{2} a_{-1} b_{0} b_{-1} \\
& +2 k^{2} a_{-1} b_{0} b_{-1} \text {, } \\
& R_{-3}=c^{2} a_{-1} b_{-1}^{2}+k^{2} a_{-1} b_{-1}^{2}+\frac{1}{2} c k a_{-1}^{2} b_{-1} \text {. }
\end{aligned}
$$


Solving this system of algebraic equations by using Maple, we get the following results:

$$
\begin{gathered}
a_{1}=0, \quad a_{0}=\mp \frac{6 k^{2} b_{0}}{\sqrt{-k^{2}-1}}, \quad a_{-1}=0, \\
b_{1}=\frac{b_{0}^{2}}{4 b_{-1}}, \quad b_{0}=b_{0}, \quad b_{-1}=b_{-1}, \\
c=\mp \sqrt{\frac{k^{2}}{-k^{2}-1}}, \quad k=k,
\end{gathered}
$$

where $b_{0}$ and $b_{-1}$ are arbitrary parameters. Substituting these results into (33), we get the following exact solution:

$$
U(\xi)=\mp \frac{6 k^{2} b_{0} / \sqrt{-k^{2}-1}}{\left(b_{0}^{2} / 4 b_{-1}\right) \exp (\xi)+b_{0}+b_{-1} \exp (-\xi)},
$$

where $b_{0}$ and $b_{1}$ are arbitrary parameters and $\xi=\left(k x^{\alpha} / \Gamma(1+\right.$ $\alpha) \mp \sqrt{k^{2} /\left(-k^{2}-1\right)}\left(t^{\alpha} / \Gamma(1+\alpha)\right)$.

Finally, if we take $b_{-1}=1$ and $b_{0}=2$, (37) becomes

$$
\begin{aligned}
& u(x, t) \\
& =\mp \frac{6 k^{2}}{\sqrt{-k^{2}-1}} \\
& \times \frac{1}{1+\cosh \left(\left(k x^{\alpha} / \Gamma(1+\alpha)\right) \mp \sqrt{k^{2} /\left(-k^{2}-1\right)}\left(t^{\alpha} / \Gamma(1+\alpha)\right)\right)}
\end{aligned}
$$

and we obtain the hyperbolic function solution of the spacetime fractional symmetric regularized long wave (SRLW) equation. Comparing our result to the results in [46], it can be seen that our solution has never been obtained.

4.3. Exact Solutions by $\left(G^{\prime} / G\right)$-Expansion Method. Recently, Zayed et al. [47] obtained solitary wave solutions to SRLW equation by means of improved $\left(G^{\prime} / G\right)$-expansion method. But they applied this method to (22). Namely, they took the constants of integration as zero.

In our study, we integrate (21) twice with respect to $\xi$ and we get

$$
\left(c^{2}+k^{2}\right) U+c k \frac{U^{2}}{2}+c^{2} k^{2} U^{\prime \prime}+\xi_{0} U+\xi_{1}=0,
$$

where $\xi_{0}$ and $\xi_{1}$ are constants of integration.

Use ansatz (39), for the linear term of highest order $U^{\prime \prime}$ with the highest order nonlinear term $U^{2}$. By simple calculation, balancing $U^{\prime \prime}$ with $U^{2}$ in (39) gives

$$
m+2=2 m
$$

so that

$$
m=2 .
$$

Suppose that the solutions of (41) can be expressed by a polynomial in $\left(G^{\prime} / G\right)$ as follows:

$$
U(\xi)=a_{0}+a_{1}\left(\frac{G^{\prime}}{G}\right)+a_{2}\left(\frac{G^{\prime}}{G}\right)^{2}, \quad a_{2} \neq 0 .
$$

By using (17) and (42) we have

$$
\begin{aligned}
U^{\prime \prime}(\xi)= & 6 b_{2}\left(\frac{G^{\prime}}{G}\right)^{4}+\left(2 b_{1}+10 b_{2} \lambda\right)\left(\frac{G^{\prime}}{G}\right)^{3} \\
& +\left(8 b_{2} \mu+3 b_{1} \lambda+4 b_{2} \lambda^{2}\right)\left(\frac{G^{\prime}}{G}\right)^{2} \\
& +\left(6 b_{2} \lambda \mu+2 b_{1} \mu+b_{1} \lambda^{2}\right)\left(\frac{G^{\prime}}{G}\right) \\
& +2 b_{2} \mu^{2}+b_{1} \lambda \mu, \\
U^{2}(\xi)= & b_{2}^{2}\left(\frac{G^{\prime}}{G}\right)^{4}+2 b_{1} b_{2}\left(\frac{G^{\prime}}{G}\right)^{3}+2 b_{0} b_{2}\left(\frac{G^{\prime}}{G}\right)^{2} \\
& +b_{1}^{2}\left(\frac{G^{\prime}}{G}\right)^{2}+2 b_{0} b_{1}\left(\frac{G^{\prime}}{G}\right)+b_{0}^{2} .
\end{aligned}
$$

Substituting (42) and (43) into (39), collecting the coefficients of $\left(G^{\prime} / G\right)^{i}(i=0, \ldots, 4)$, and setting it to zero, we obtain the following system:

$$
\begin{aligned}
& -\frac{1}{2} c k a_{2}^{2}+6 c^{2} k^{2} a_{2}=0, \\
& 2 c^{2} k^{2} a_{1}-c k a_{1} a_{2}+10 c^{2} k^{2} a_{2} \lambda=0, \\
& -\frac{1}{2} c k a_{1}^{2}-c k a_{0} a_{2}+8 c^{2} k^{2} a_{2} \mu+3 c^{2} k^{2} a_{1} \lambda \\
& +\xi_{0} a_{2}+4 c^{2} k^{2} a_{2} \lambda^{2}+k^{2} a_{2}+c^{2} a_{2}=0, \\
& -c k a_{0} a_{1}+c^{2} k^{2} a_{1} \lambda^{2}+k^{2} a_{1}+\xi_{0} a_{1}+6 c^{2} k^{2} a_{2} \lambda \mu \\
& +c^{2} a_{1}+2 c^{2} k^{2} a_{1} \mu=0, \\
& -\frac{1}{2} c k a_{0}^{2}+2 c^{2} k^{2} a_{2} \mu^{2}+c^{2} a_{0}+\xi_{0} a_{0}+k^{2} a_{0} \\
& +c^{2} k^{2} a_{1} \lambda \mu+\xi_{1}=0 .
\end{aligned}
$$

Solving this system by using Maple gives

$$
\begin{gathered}
a_{0}=\frac{\xi_{0}+c^{2}+k^{2}+c^{2} k^{2} \lambda^{2}+8 c^{2} k^{2} \mu}{c k}, \quad a_{1}=12 c k \lambda, \\
a_{2}=12 c k, \quad c=c, \\
k=k, \quad \xi_{0}=\xi_{0}, \\
\xi_{1}=\left(-2 c^{2} k^{2}-8 c^{4} k^{4} \lambda^{2} \mu+16 c^{4} k^{4} \mu^{2}+c^{4} k^{4} \lambda^{4}-k^{4}\right. \\
\left.-c^{4}-2 k^{2} \xi_{0}-2 c^{2} \xi_{0}-\xi_{0}^{2}\right)(2 c k)^{-1},
\end{gathered}
$$

where $\lambda, \mu, \xi_{0}$, and $\xi_{1}$ are arbitrary constants. 
By using (42), expression (45) can be written as

$$
\begin{aligned}
U(\xi)= & \frac{\xi_{0}+c^{2}+k^{2}+c^{2} k^{2} \lambda^{2}+8 c^{2} k^{2} \mu}{c k} \\
& +12 c k \lambda\left(\frac{G^{\prime}}{G}\right)+12 c k\left(\frac{G^{\prime}}{G}\right)^{2} .
\end{aligned}
$$

Substituting general solutions of (17) into (46) we have three types of travelling wave solutions of space-time fractional symmetric regularized long wave (SRLW) equation. These are the following.

When $\lambda^{2}-4 \mu>0$,

$$
\begin{aligned}
& U_{1}(\xi) \\
& =\frac{\xi_{0}+k^{2}+c^{2}}{c k}-2 c k\left(\lambda^{2}-4 \mu\right)+3 c k\left(\lambda^{2}-4 \mu\right) \\
& \quad \times\left(\frac{C_{1} \sinh (1 / 2) \sqrt{\lambda^{2}-4 \mu} \xi+C_{2} \cosh (1 / 2) \sqrt{\lambda^{2}-4 \mu} \xi}{C_{1} \cosh (1 / 2) \sqrt{\lambda^{2}-4 \mu} \xi+C_{2} \sinh (1 / 2) \sqrt{\lambda^{2}-4 \mu} \xi}\right),
\end{aligned}
$$

where $\xi=\left(k x^{\alpha} / \Gamma(1+\alpha)\right)+\left(c t^{\alpha} / \Gamma(1+\alpha)\right)$.

When $\lambda^{2}-4 \mu<0$,

$$
\begin{aligned}
& U_{2}(\xi) \\
& =\frac{\xi_{0}+k^{2}+c^{2}}{c k}-2 c k\left(\lambda^{2}-4 \mu\right)+3 c k\left(4 \mu-\lambda^{2}\right) \\
& \quad \times\left(\frac{-C_{1} \sin (1 / 2) \sqrt{4 \mu-\lambda^{2}} \xi+C_{2} \cos (1 / 2) \sqrt{4 \mu-\lambda^{2}} \xi}{C_{1} \cos (1 / 2) \sqrt{4 \mu-\lambda^{2}} \xi+C_{2} \sin (1 / 2) \sqrt{4 \mu-\lambda^{2}} \xi}\right)^{2},
\end{aligned}
$$

where $\xi=\left(k x^{\alpha} / \Gamma(1+\alpha)\right)+\left(c t^{\alpha} / \Gamma(1+\alpha)\right)$.

When $\lambda^{2}-4 \mu=0$,

$$
\begin{aligned}
u_{3}(x, t)= & \frac{\xi_{0}+k^{2}+c^{2}}{c k}+12 c k \\
& \times\left(\frac{C_{2}}{C_{1}+C_{2}\left(\left(k x^{\alpha} / \Gamma(1+\alpha)\right)+\left(c t^{\alpha} / \Gamma(1+\alpha)\right)\right)}\right)^{2} .
\end{aligned}
$$

In particular, if $C_{1} \neq 0, C_{2}=0, \lambda>0, \mu=0$, then $U_{1}$ and $U_{2}$ become

$$
\begin{aligned}
u_{1}(x, t)= & \frac{\xi_{0}+k^{2}+c^{2}}{c k}-2 c k \lambda^{2} \\
& +3 c k \lambda^{2} \tanh ^{2}\left\{\frac{\lambda}{2}\left(\frac{k x^{\alpha}}{\Gamma(1+\alpha)}+\frac{c t^{\alpha}}{\Gamma(1+\alpha)}\right)\right\} .
\end{aligned}
$$

Comparing our results to Zayed's results [47], it can be seen that these results are new.

\section{Conclusion}

In this paper, the functional variable method, the expfunction method, and $\left(G^{\prime} / G\right)$-expansion method have been successfully employed to obtain solution of the space-time fractional symmetric regularized long wave (SRLW) equation. These solutions include the generalized hyperbolic function solutions, generalized trigonometric function solutions, and rational function solutions, which may be very useful to understand the nonlinear FDEs and our result can turn into hyperbolic solution when suitable parameters are chosen. To the best of our knowledge, the solutions obtained in this paper have not been reported in literature. Maple has been used for programming and computations in this work.

\section{Conflict of Interests}

The authors declare that there is no conflict of interests regarding the publication of this paper.

\section{References}

[1] K. S. Miller and B. Ross, An Introduction to the Fractional Calculus and Fractional Differential Equations, John Wiley \& Sons, New York, NY. USA, 1993.

[2] I. Podlubny, Fractional Differential Equations, vol. 198 of Mathematics in Science and Engineering, Academic Press, San Diego, Calif, USA, 1999.

[3] A. A. Kilbas, H. M. Srivastava, and J. J. Trujillo, Theory and Applications of Fractional Differential Equations, NorthHolland Mathematics Studies, Elsevier Science B.V., Amsterdam, Netherlands, 2006.

[4] H. Sun and W. Chen, "Fractal derivative multi-scale model of fluid particle transverse accelerations in fully developed turbulence," Science in China, Series E: Technological Sciences, vol. 52, no. 3, pp. 680-683, 2009.

[5] W. Chen and H. Sun, "Multiscale statistical model of fullydeveloped turbulence particle accelerations," Modern Physics Letters B, vol. 23, no. 3, pp. 449-452, 2009.

[6] J. Cresson, "Scale calculus and the Schrödinger equation," Journal of Mathematical Physics, vol. 44, no. 11, pp. 4907-4938, 2003.

[7] J. Cresson, "Non-differentiable variational principles," Journal of Mathematical Analysis and Applications, vol. 307, no. 1, pp. 48-64, 2005.

[8] S. G. Samko, A. A. Kilbas, and O. I. Marichev, Fractional Integrals and Derivatives: Theory and Applications, Gordon and Breach Science, Yverdon, Switzerland, 1993.

[9] M. Caputo, "Linear models of dissipation whose Q is almost frequency independent II," Geophysical Journal International, vol. 13, no. 5, pp. 529-539, 1967.

[10] B. Zheng, “ $\left(G^{\prime} / G\right)$-expansion method for solving fractional partial differential equations in the theory of mathematical physics," Communications in Theoretical Physics, vol. 58, no. 5, pp. 623-630, 2012.

[11] K. A. Gepreel and S. Omran, "Exact solutions for nonlinear partial fractional differential equations," Chinese Physics B, vol. 21, no. 11, Article ID 110204, 2012. 
[12] A. Bekir and Ö. Güner, "Exact solutions of nonlinear fractional differential equations by $\left(G^{\prime} / G\right)$-expansion method," Chinese Physics B, vol. 22, no. 11, Article ID 110202, 2013.

[13] N. Shang and B. Zheng, "Exact solutions for three fractional partial differential equations by the $\left(G^{\prime} / G\right)$ method," IAENG International Journal of Applied Mathematics, vol. 43, no. 3, pp. 114-119, 2013.

[14] S. Zhang, Q.-A. Zong, D. Liu, and Q. Gao, "A generalized expfunction method for fractional riccati differential equations," Communications in Fractional Calculus, vol. 1, no. 1, pp. 48-51, 2010.

[15] A. Bekir, Ö. Güner, and A. C. Cevikel, "Fractional complex transform and exp-function methods for fractional differential equations," Abstract and Applied Analysis, vol. 2013, Article ID 426462, 8 pages, 2013.

[16] B. Zheng, "Exp-function method for solving fractional partial differential equations," The Scientific World Journal, vol. 2013, Article ID 465723, 8 pages, 2013.

[17] B. Lu, "The first integral method for some time fractional differential equations," Journal of Mathematical Analysis and Applications, vol. 395, no. 2, pp. 684-693, 2012.

[18] M. Eslami, B. F. Vajargah, M. Mirzazadeh, and A. Biswas, "Application of first integral method to fractional partial differential equations," Indian Journal of Physics, vol. 88, no. 2, pp. 177-184, 2014.

[19] S. Zhang and H.-Q. Zhang, "Fractional sub-equation method and its applications to nonlinear fractional PDEs," Physics Letters A, vol. 375, no. 7, pp. 1069-1073, 2011.

[20] B. Zheng and C. Wen, "Exact solutions for fractional partial differential equations by a new fractional sub-equation method," Advances in Difference Equations, vol. 2013, article 199, 2013.

[21] J. F. Alzaidy, "Fractional sub-equation method and its applications to the space-time fractional differential equations in mathematical physics," British Journal of Mathematics \& Computer Science, vol. 3, no. 2, pp. 153-163, 2013.

[22] H. Jafari, H. Tajadodi, N. Kadkhoda, and D. Baleanu, "Fractional subequation method for Cahn-Hilliard and KleinGordon equations," Abstract and Applied Analysis, vol. 2013, Article ID 587179, 5 pages, 2013.

[23] A. Nazarzadeh, M. Eslami, and M. Mirzazadeh, "Exact solutions of some nonlinear partial differential equations using functional variable method," Pramana-Journal of Physics, vol. 81, no. 2, pp. 225-236, 2013.

[24] H. Bulut, M. Baskonus H, and Y. Pandir, "The modified trial equation method for fractional wave equation and time fractional generalized burgers equation," Abstract and Applied Analysis, vol. 2013, Article ID 636802, 8 pages, 2013.

[25] Y. Pandir, Y. Gurefe, and E. Misirli, "New exact solutions of the time-fractional nonlinear dispersive KdV equation," International Journal of Modeling and Optimization, vol. 3, no. 4, pp. 349-352, 2013.

[26] N. Taghizadeh, M. Mirzazadeh, M. Rahimian, and M. Akbari, "Application of the simplest equation method to some timefractional partial differential equations," Ain Shams Engineering Journal, vol. 4, no. 4, pp. 897-902, 2013.

[27] G. Jumarie, "Modified Riemann-Liouville derivative and fractional Taylor series of nondifferentiable functions further results," Computers \& Mathematics with Applications, vol. 51, no. 9-10, pp. 1367-1376, 2006.
[28] G. Jumarie, "Table of some basic fractional calculus formulae derived from a modified Riemann-Liouville derivative for nondifferentiable functions," Applied Mathematics Letters, vol. 22, no. 3, pp. 378-385, 2009.

[29] G. Jumarie, "Laplace's transform of fractional order via the Mittag-Leffler function and modified Riemann-Liouville derivative," Applied Mathematics Letters, vol. 22, no. 11, pp. 16591664,2009

[30] Z. Li and J. He, "Fractional complex transform for fractional differential equations," Mathematical \& Computational Applications, vol. 15, no. 5, pp. 970-973, 2010.

[31] Z. B. Li and J. H. He, "Application of the fractional complex transform to fractional differential equations," Nonlinear Science Letters A: Mathematics, Physics and Mechanics, vol. 2, pp. 121-126, 2011.

[32] J. He, S. K. Elagan, and Z. B. Li, "Geometrical explanation of the fractional complex transform and derivative chain rule for fractional calculus," Physics Letters A, vol. 376, no. 4, pp. 257$259,2012$.

[33] A. Zerarka, S. Ouamane, and A. Attaf, "On the functional variable method for finding exact solutions to a class of wave equations," Applied Mathematics and Computation, vol. 217, no. 7, pp. 2897-2904, 2010.

[34] J. He and M. A. Abdou, "New periodic solutions for nonlinear evolution equations using Exp-function method," Chaos, Solitons \& Fractals, vol. 34, no. 5, pp. 1421-1429, 2007.

[35] J. He and X. Wu, "Exp-function method for nonlinear wave equations," Chaos, Solitons and Fractals, vol. 30, no. 3, pp. 700708, 2006.

[36] A. Bekir and A. Boz, "Application of He's exp-function method for nonlinear evolution equations," Computers \& Mathematics with Applications, vol. 58, no. 11-12, pp. 2286-2293, 2009.

[37] A. Ebaid, "An improvement on the Exp-function method when balancing the highest order linear and nonlinear terms," Journal of Mathematical Analysis and Applications, vol. 392, no. 1, pp. 15, 2012.

[38] I. Aslan, "On the application of the Exp-function method to the KP equation for $N$-soliton solutions," Applied Mathematics and Computation, vol. 219, no. 6, pp. 2825-2828, 2012.

[39] S. Yu, " $N$-soliton solutions of the KP equation by Exp-function method," Applied Mathematics and Computation, vol. 219, no. 8, pp. 3420-3424, 2012.

[40] S. Zhang and H. Zhang, "An Exp-function method for a new $\$ N \$$-soliton solutions with arbitrary functions of a $\$(2+1) \$$ dimensional vcBK system," Computers \& Mathematics with Applications, vol. 61, no. 8, pp. 1923-1930, 2011.

[41] M. Wang, X. Li, and J. Zhang, “The $\left(G^{\hat{E}} / G\right)$-expansion method and travelling wave solutions of nonlinear evolution equations in mathematical physics," Physics Letters. A, vol. 372, no. 4, pp. 417-423, 2008.

[42] A. Bekir, "Application of the $\left(G^{\hat{\mathrm{E}} z} / G\right)$-expansion method for nonlinear evolution equations," Physics Letters A, vol. 372, no. 19, pp. 3400-3406, 2008.

[43] H. Jafari, N. Kadkhoda, and A. Biswas, "The $G^{\hat{\mathrm{E}} \dot{ }} / G$-expansion method for solutions of evolution equations from isothermal magnetostatic atmospheres," Journal of King Saud UniversityScience, vol. 25, no. 1, pp. 57-62, 2013.

[44] J. F. Alzaidy, "The fractional sub-equation method and exact analytical solutions for some nonlinear fractional PDEs," American Journal of Mathematical Analysis, vol. 1, no. 1, pp. 14-19, 2013. 
[45] H. Jafari, A. Borhanifar, and S. A. Karimi, "New solitary wave solutions for generalized regularized long-wave equation," International Journal of Computer Mathematics, vol. 87, no. 1-3, pp. 509-514, 2010.

[46] F. Xu, "Application of Exp-function method to symmetric regularized long wave (SRLW) equation," Physics Letters A, vol. 372, no. 3, pp. 252-257, 2008.

[47] E. M. E. Zayed, Y. A. Amer, and R. M. A. Shohib, "Exact traveling wave solutions for nonlinear fractional partial differential equations using the improved ( $\left.G^{\prime} / G\right)$-expansion method," International Journal of Engineering and Applied Science, vol. 7, pp. 18-31, 2014. 


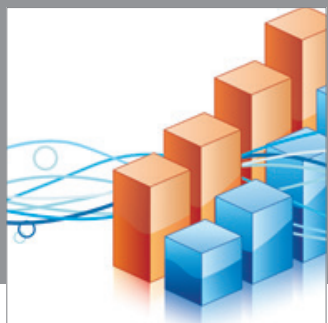

Advances in

Operations Research

mansans

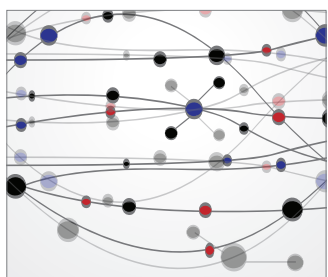

The Scientific World Journal
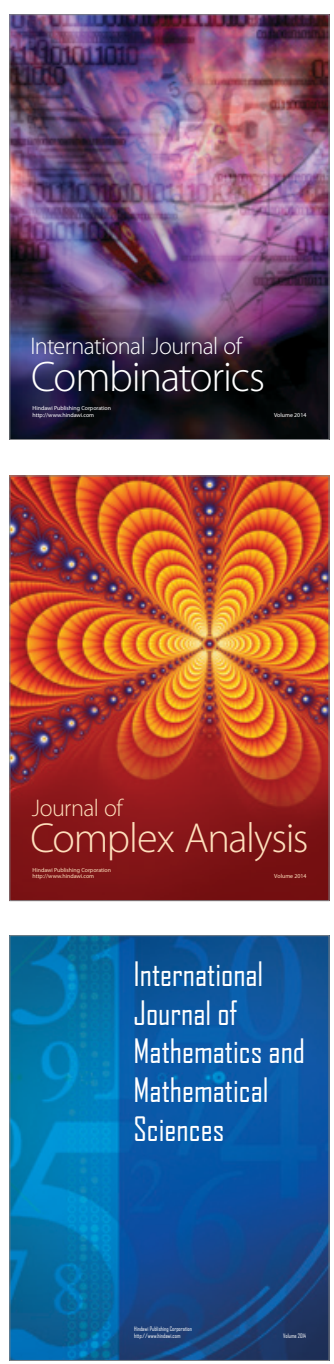
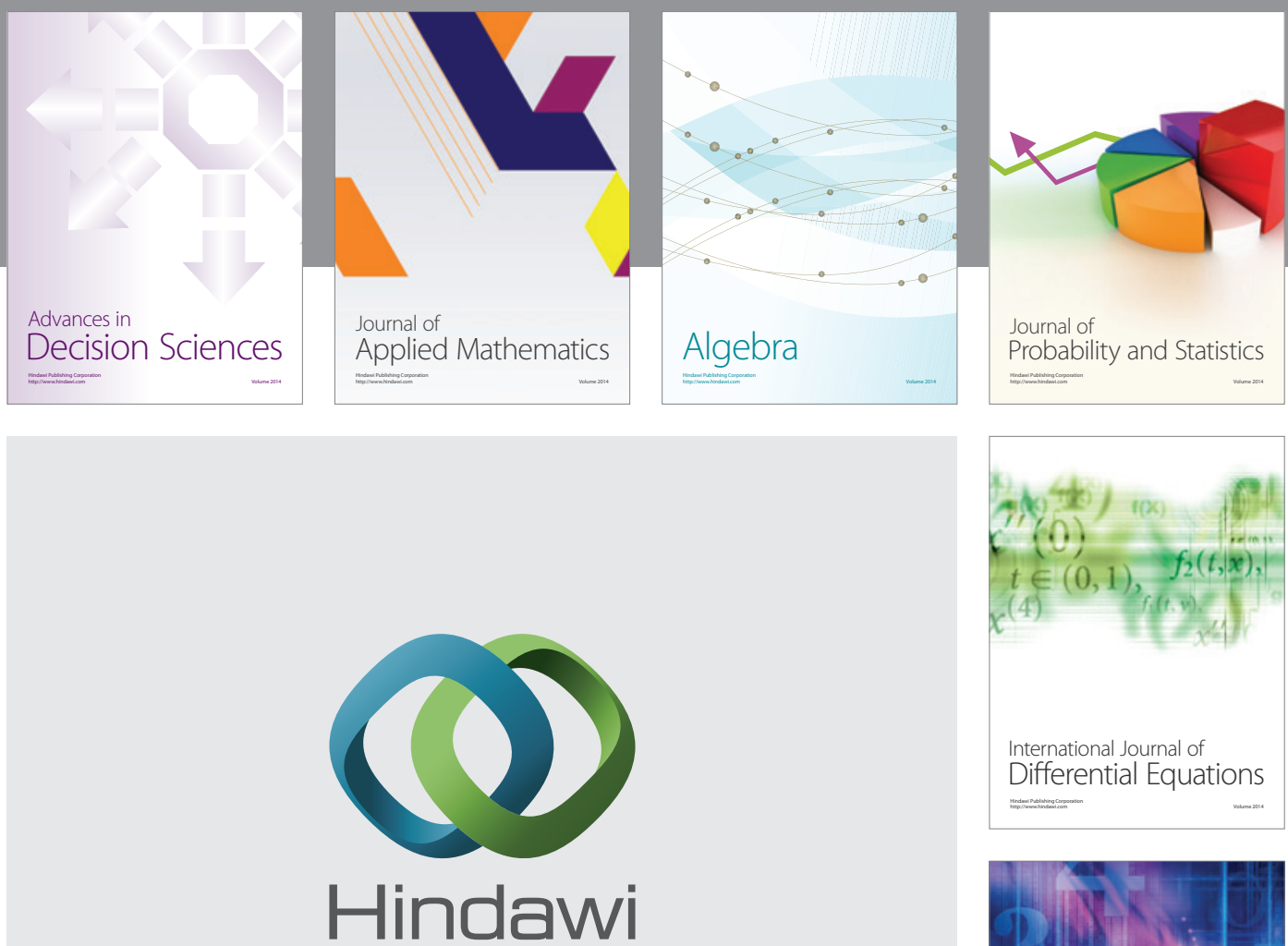

Submit your manuscripts at http://www.hindawi.com
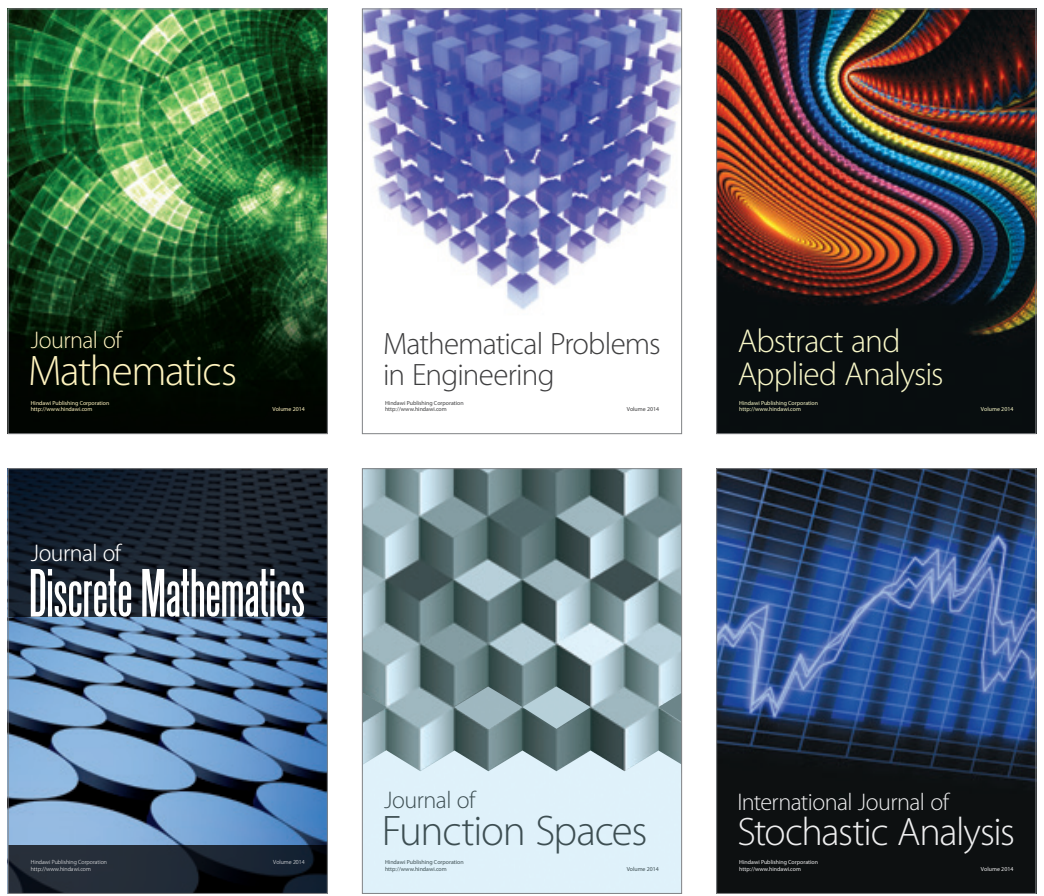

Journal of

Function Spaces

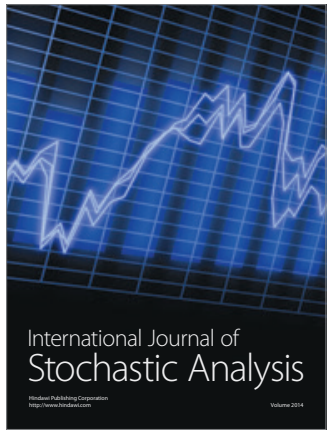

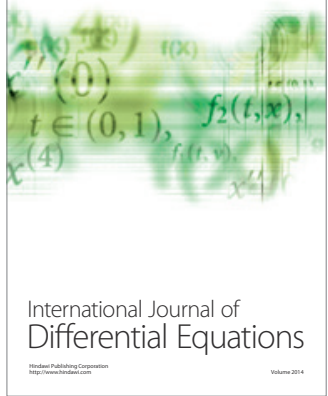
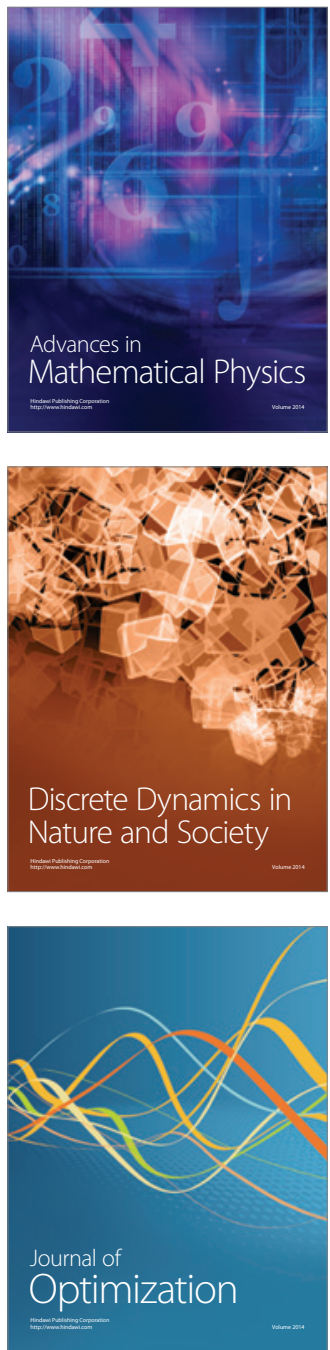